\author{
Puteri S. Abdul-Rahman' \\ Boon-Kiong Lim² \\ Onn H. Hashim ${ }^{1}$ \\ ${ }^{1}$ Department of \\ Molecular Medicine, \\ Faculty of Medicine, \\ University of Malaya, \\ Kuala Lumpur, Malaysia \\ ${ }^{2}$ Department of Obstetrics \\ and Gynaecology, \\ Faculty of Medicine, \\ University of Malaya, \\ Kuala Lumpur, Malaysia
}

Received October 1, 2006

Revised January 28, 2007

Accepted January 31, 2007
Research Article

\section{Expression of high-abundance proteins in sera of patients with endometrial and cervical cancers: Analysis using 2-DE with silver staining and lectin detection methods}

The expression of high-abundance serum proteins in newly diagnosed patients with endometrial adenocarcinoma (EACa), squamous cell cervical carcinoma (SCCa) and cervical adenocarcinoma (ACCa), relative to control female subjects, was analyzed by subjecting serum samples to 2-DE followed by image analysis of the silver-stained protein profiles. The three cohorts of cancer patients demonstrated different altered expression of serum highabundance proteins compared to negative control women. The expression of $\alpha_{1}$-antitrypsin, $\alpha_{1}$-B glycoprotein, cleaved high-molecular-weight kininogen (light chain) and antithrombin III were consistently altered in all the patients. However, clusterin was upregulated only in the patients with EACa, while those with SCCa and ACCa were typically characterized by the upregulated expression of zinc $\alpha$-2-glycoprotein. The aberrant expression of selective serum proteins in the various cohorts of cancer patients was validated by competitive ELISA as well as by lectin detection. Analysis by using the champedak galactose binding lectin further highlighted an unidentified protein that may be differently glycosylated in the sera of the EACa patients that were studied.

\section{Keywords: \\ Cervical cancer / Endometrial cancer / Lectin / O-Glycans / Proteomics}

DOI 10.1002/elps.200600629

\section{Introduction}

Other than ovarian carcinoma, the two most common gynecological malignancies that affect women worldwide are cancers of the endometrium and the cervix. Endometrial adenocarcinoma (EACa) is the most common type of endometrial cancer while the two types of cervical cancer are squamous cell cervical carcinoma (SCCa) and cervical adenocarcinoma (ACCa), with SCCa accounting for up to 60 $80 \%$ of the cases [1].

Correspondence: Professor Onn H. Hashim, Department of Molecular Medicine, Faculty of Medicine, University of Malaya, 50603 Kuala Lumpur, Malaysia

E-mail: onnhashim@um.edu.my

Abbreviations: AAT, $\alpha_{1}$-antitrypsin; ABG, $\alpha_{1}$-B glycoprotein; ACCa, cervical adenocarcinoma; ACT, $\alpha_{1}$-antichymotrypsin; AHS, $\alpha_{2}$-HS glycoprotein; ATR, antithrombin III; $\mathbf{B r C a}$, breast carcinoma; CGB lectin, champedak galactose binding lectin; CLU, clusterin; EACa, endometrial adenocarcinoma; HAP, haptoglobin ( $\beta$-chain); HPX, hemopexin; KNG, cleaved high-molecular-weight kininogen (light chain); LRG, leucine-rich glycoprotein; NPCa, nasopharyngeal carcinoma; SCCa, squamous cell cervical carcinoma; ZAG, zinc $\alpha$-2-glycoprotein
At present, serological diagnoses of gynecological cancers are constrained to the analyses of CA 125, CA 19.9 and SCC antigen tumor markers [2]. These markers are limited in specificities and not readily detectable in early stages of the disease. For example, CA 125 is the common tumor marker used for diagnoses of ovarian and endometrial cancers [3, 4]. However, its presence along with CA 19.9 has also been reported to be significantly higher in patients with ACCa compared to SCCa patients [5]. On the other hand, SCC antigen is adept for detection of SCCa but also serves as a tumour marker for more advanced squamous cell tumors of the lungs and oropharynx [6-8].

The search for new serological tumour markers is gaining momentum through the use of proteomics technologies $[9,10]$. Instead of relying on single tumor markers as currently practised, proteomics approaches have unravelled series of newly detected biomarkers, which when analyzed simultaneously may be able to discriminate different types of cancers, their stages or monitor the progress of cancer treatment towards patient's convalescence. Considerable proteomics analyses have been performed on albumin and Ig depleted serum samples. However, albumin and Igs bind to a number of serum proteins including those that have been clinically or experimentally used as biomarkers [11]. Thus, 
the removal of albumin and Ig's may indirectly contribute to the differential expression of proteins that was detected.

Previous studies using whole unfractionated serum samples of rats and humans have demonstrated that even high-abundance proteins could be the markers for disease states [12-15]. By using 2-DE and image analysis, we have reported the aberrant expression of high-abundance acutephase serum proteins in patients with cancers of the breast (breast carcinoma; BrCa) and nasopharynx (nasopharyngeal carcinoma; NPCa). While patients with $\mathrm{BrCa}$ demonstrated different altered expression of serum $\alpha_{1}$-antichymotrypsin (ACT), clusterin (CLU) and complement factor B [13], ceruloplasmin was the sole high-abundance protein that was upregulated in the sera of NPCa patients [14]. However, the question of whether the altered patterns of expression of the serum proteins is truly unique for the respective cancers could only be resolved if similar proteomics analyses were broadened to patients with other types of cancer.

In the present study, analysis of the expression of highabundance serum proteins has been extended to newly diagnosed patients afflicted with EACa, SCCa and ACCa. In addition, we have also generated 2-DE profiles of O-glycosylated serum proteins of the patients by using the champedak galactose binding (CGB) lectin probe and compared them with that obtained from normal negative control women.

\section{Materials and methods}

\subsection{Serum samples}

Serum samples were obtained from patients newly confirmed with gynecological malignancies at the Gynecology Clinic, University of Malaya Medical Center, Kuala Lumpur, prior to treatment or surgery. The patients (EACa $(n=12)$, SCCa $(n=10)$ and ACCa $(n=7))$, with ages ranging from 35 to 65 years, were histopathologically confirmed for stage $1 \mathrm{~A}$ or 1B carcinoma. Control sera $(n=13)$ were obtained from normal healthy women volunteers of the same range of age. Samples were obtained with patient's consent and approval granted by the Ethical Committee (Institutional Review Board) of the Medical Centre in accordance to the ICH GCP guideline and the declaration of Helsinki. All the samples were subjected to similar treatment and kept in aliquots of $100 \mu \mathrm{L}$ at $-80^{\circ} \mathrm{C}$.

\subsection{2-DE}

2-DE was performed as previously reported [13]. Briefly, $7 \mu \mathrm{L}$ of unfractionated whole human serum sample $(450 \mu \mathrm{g}$ of protein) was subjected to IEF in $11 \mathrm{~cm}$ rehydrated precast Immobiline Drystrips at $\mathrm{pH} 4-7$ (GE Healthcare BioSciences, Uppsala, Sweden). For the second dimension, focused samples in the strips were subjected to electrophoresis using $8-18 \%$ gradient polyacrylamide gel in the presence of SDS. All the samples were analyzed in duplicate.

\subsection{Gel staining}

The 2-DE gels were developed by silver staining as described by Heukeshoven and Dernick [16]. For mass spectrometric analysis, gels were stained using Coomassie colloidal stain. The Coomassie stain was prepared by dissolving $0.1 \% \mathrm{w} / \mathrm{v}$ Phast Blue R350 (GE Healthcare BioSciences) in 10\% acetic acid solution that was preheated to $90^{\circ} \mathrm{C}$. Gels were stained on a rocking platform for a period of $30 \mathrm{~min}$ followed by destaining with $10 \%$ acetic acid solution.

\section{$2.4 \mathrm{MS}$}

Protein spots were initially identified by comparing highly resolved serum protein profiles with the Swiss ExPASy standard plasma protein reference [17]. 2-DE gel plugs of spots of proteins of interest were sent for MS analysis using MALDI-TOF at the Biomolecular Research Facility, University of Newcastle, Australia. Samples that were not positively identified were then subjected to MS/MS analysis at the Australian Proteomic Analysis Facility (APAF), Macquarie University, Sydney, Australia. Gel plugs were kept in fresh eppendorf tubes containing small volumes of Milli-Q water to ensure that they remained hydrated prior to analysis.

\subsection{Database searches}

Identities of protein spots of interest were initially determined by the Biomolecular Research Facility, University of Newcastle, Australia. The identities were reconfirmed using MASCOT program (http://www.matrixscience.com) by submitting the mass list data to the peptide mass fingerprint search. The following parameters were used in the searches: trypsin digest (allowing one missed cleavage); species, Homo sapiens; mass value, monoisotopic; peptide mass tolerance, $\pm 0.1 \mathrm{Da}$; peptide charge state, $1+$ and against the NCBInr database. The same parameters were used in the MASCOT ions search for the MS/MS data, except peptide mass and fragment mass tolerance were set at $\pm 300 \mathrm{ppm}$ and $\pm 0.6 \mathrm{Da}$, respectively.

\subsection{Western blotting and lectin detection}

The 2-DE gels were transferred electrophoretically onto an NC membrane $(0.45 \mu \mathrm{m})$ using a NovaBlot Kit of the Multiphor II Electrophoresis System (GE Healthcare BioSciences). Blotting was performed at $0.8 \mathrm{~mA} / \mathrm{cm}^{2}$ for $2 \mathrm{~h}$. Detection of transferred $O$-glycosylated serum proteins was performed using CGB lectin which was conjugated to horseradish peroxidase (HRP). The lectin was chosen based on its specific interaction with O-glycans of glycoproteins [18]. Purity and specificity of the lectin were confirmed using previously described biochemical and immunochemical analyses [18-20]. Blots were developed using 3,3'-diaminobenzidine (BioRad Laboratories, Hercules, CA, USA). Reac- 
tion was stopped by washing the membrane twice with distilled water. The blots were air dried prior to densitometric scanning and image analysis.

\subsection{Image analysis}

The silver-stained gels and lectin blots were scanned using a densitometer (Imaging Densitometer GS690, BioRad Laboratories). Protein spots were analysed in terms of volume performed using the Image Master 2D-Platinum software, version 5.0 (GE Healthcare BioSciences). The background was subtracted and analysis was restricted to 12 clusters of protein spots with $M_{\mathrm{r}} \geq 30000$, i.e. $\alpha_{1}$-antitrypsin (AAT), $\alpha_{2}$-HS glycoprotein (AHS), $\alpha_{1}$-B glycoprotein (ABG), haptoglobin $\beta$-chain (HAP), ACT, CLU, anti-thrombin III (ATR), leucine-rich glycoprotein (LRG), cleaved high-molecular-weight kininogen light chain (KNG) and zinc $\alpha$-2-glycoprotein (ZAG) for the 2-DE protein profiles that were generated by silver staining. Analysis of the 2-DE protein profiles developed by interaction with lectin was performed on ACT, AHS, LRG, hemopexin (HPX), KNG and unidentified proteins LDP1, LDP2 and LDP3. Albumin, serum polypeptides having idiotypic and/or allotypic variations (such as the heavy and light chains of all the isotypes of Igs and the $\alpha$ chains of HAP) and the low $M_{r}$ protein spots, the majority of which were not well resolved under the settings of our experiments, were not assessed. The percentage volume contribution refers to the volume percentage of a protein taken against the total spot volume of all the proteins including the unresolved peptides in each gel.

\subsection{Competitive ELISA}

Competitive ELISA was performed as previously described [13]. The levels of AAT, ATR, CLU and ABG in serum samples were reflected by their ability to inhibit the specific binding of antisera to the respective serum proteins. Primary antisera against AAT and ATR were obtained from the Binding Site (Birmingham, UK) while those against CLU and ABG were purchased from Upstate Biotechnology (Lake Placid, USA) and Aviva Systems Biology (San Diego, USA), respectively. Assay was not performed for LRG and ZAG as antisera to the serum proteins were not available commercially. HRP-conjugated secondary antisera comprising donkey anti-sheep IgG, goat anti-mouse IgG and goat anti-rabbit IgG, were obtained from the Binding Site, Sigma-Aldrich (St. Louis, USA) and BioRad Laboratories, respectively. Enzyme activity was revealed by the addition of $3,3^{\prime}, 5,5^{\prime}$-tetramethylbenzidine (Pierce, Rockford, IL, USA). The amount of specific proteins in the test sera is proportional to the percentage inhibition of substrate hydrolysis.

\subsection{Statistical analysis}

All the values are presented as mean \pm SD. The Student's $t$-test was used to analyze the significance of differences be- tween normal subjects and patients. A $p$-value of less than 0.05 was considered significant.

\section{Results}

When unfractionated whole serum samples from newly diagnosed patients with EACa $(n=12)$, SCCa $(n=10)$ and ACCa $(n=7)$ as well as normal healthy women $(n=13)$ were subjected to 2-DE and silver staining, high-resolution serum protein profiles were obtained (Fig. 1). Among the highabundance proteins that were well resolved in the various protein profiles were AAT, ABG, ACT, AHS, ATR, CLU, HAP, KNG, LRG and ZAG.

The identities of AAT, ABG, ACT, AHS and HAP were confirmed by subjecting their spot clusters to in gel trypsin digestion and MS, as previously reported [13]. In cases of the spot clusters of ATR, CLU, LRG and ZNG, initial identification was made through comparison with the Swiss ExPASy standard plasma protein reference map [17] and this was further confirmed by subjecting the protein spot clusters to MALDI-MS analysis and database search (Table 1). KNG was identified using 1-D LC-MS/MS with nine sequences of peptides correlating to the protein.

Volume analysis of ten selective well-resolved spot clusters in the 2-DE profiles of patients, compared to that of control women, demonstrated different altered expression of serum high-abundance proteins in the various cohorts of cancer patients that were studied (Fig. 2). The upregulated expression of ABG, ATR, CLU and LRG and downregulated expression of AAT and KNG were significantly detected in the profiles of the EACa patients. The expression of serum high-abundance proteins was similar in the two cohorts of patients with SCCa and ACCa. In both these cohorts of cervical cancer patients, significant upregulated expression was detected for ABG, ATR and ZAG, while the expression of AAT and KNG was significantly lower. The expression of AHS, ACT and HAP was not significantly different between patients and control women.

For confirmation of the aberrantly expressed identified proteins in sera of the respective cancer patients, competitive ELISA was carried out by using antisera to the selective serum proteins. Table 2 summarizes of the results of the competitive ELISA as compared to the data obtained from analysis by 2-DE. Higher levels of ABG and ATR and lower levels of AAT were significantly detected in sera of all the patients (EACa $(n=12)$, SCCa $(n=10)$ and ACCa $(n=7)$ ) compared to normal controls $(n=13)$. The expression of CLU was only enhanced in the patients with EACa but not significantly different for patients with SCCa and ACCa.

The application of lectin to probe selective glycosylated polypeptides which were resolved by 2-DE allows one to validate the data obtained by silver staining as well as to screen for the possible presence of structural aberration in the oligosaccharide moieties of the serum glycoproteins. When 2-DE separated sera of patients and negative control 

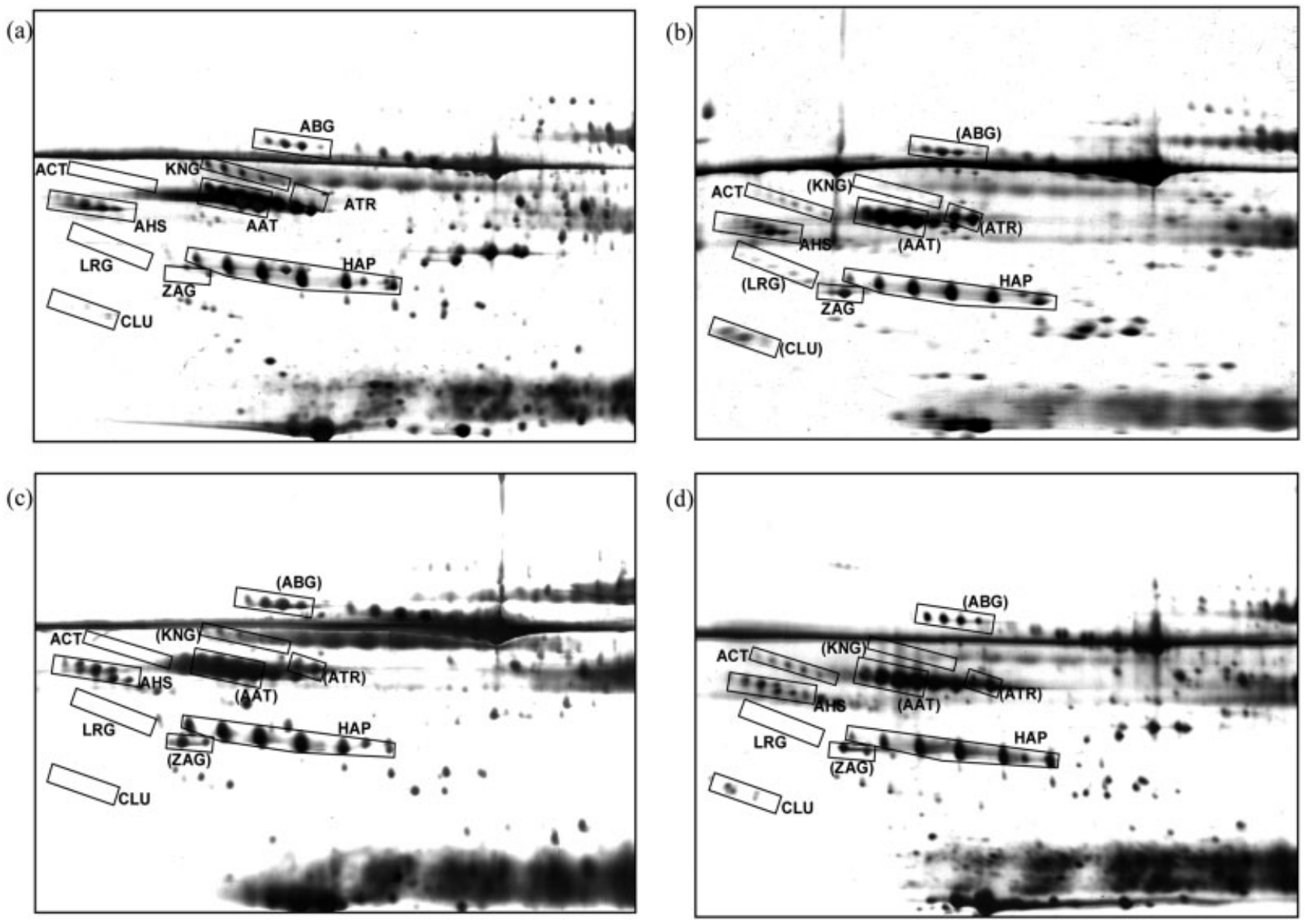

Figure 1. Typical serum protein profiles of negative control women and patients with EACa, SCCa and ACCa. Unfractionated whole serum samples of normal healthy control women were subjected to 2-DE and silver staining. (a) Typical serum protein profile of a healthy control woman. Clusters of protein spots clearly resolved include AAT, ABG, ACT, AHS, ATR, CLU, HAP, KNG, LRG and ZAG. (b-d) Typical representative unfractionated serum protein profiles of the EACa, SCCa and ACCa patients, respectively. The aberrantly expressed proteins are marked in brackets for panels (b-d). For all the panels, the acidic sides of the 2-DE gels are to the left and $M_{\mathrm{r}}$ declines from the top.

women were exposed to enzyme-conjugated champedak galactose binding (CGB) lectin, different profiles comprising only O-glycosylated peptides were obtained (Fig. 3). The spots of HPX appeared to be well resolved in the profiles with the absence of the albumin streak that usually appeared using the silver staining method. The spots of KNG, an $O$ glycosylated polypeptide, were further validated as they were recognized by the lectin. Three additional unidentified protein spot clusters that were not detected in the silver-stained profiles, termed LDP1, LDP2 and LDP3, were also observed. Comparable results were generally observed for the 2-DE $O$ glycosylated peptide profiles of the two cohorts of patients with cervical cancer and control subjects. However, EACa patients were notably different for their intense expression of LDP1.

Subjecting the 2-DE lectin-detected serum protein profiles to densitometry analysis confirmed the lowered expression of KNG in all cohorts of cancer patients and the significant enhanced expression of LDP1 in patients with EACa compared to negative control women (Fig. 4). The image analysis also detected significant reduced expression of HPX selectively in the same cohort of cancer patients. Like that observed in the silver-stained 2-DE analysis, the levels of AHS and ACT were not significantly different between patients and negative control women. However, the levels of LRG were not significantly different between all patients and negative control women, although our data obtained by silver staining demonstrate significant difference of the LRG expression in patients with EACa. The expression of LDP3 was significantly reduced in all three cohorts of cancer patients compared to the controls.

\section{Discussion}

The present study, performed by subjecting serum samples to 2-DE followed by silver staining and image analysis, demonstrated different altered levels of several high-abun- 
Table 1. Mass spectrometric identification of spot clusters from serum protein profiles

\begin{tabular}{llllll}
\hline Spot ID & $\begin{array}{l}\text { Accession } \\
\text { No. }{ }^{\text {b) }}\end{array}$ & $M_{\mathrm{r}}(\mathrm{kDa}) /$ calculated $\mathrm{p} /$ & $\begin{array}{l}\text { Number of peaks } \\
\text { matched (number } \\
\text { of peaks searched) }\end{array}$ & $\begin{array}{l}\text { MASCOT } \\
\text { score }\end{array}$ & $\begin{array}{l}\text { Sequence } \\
\text { percent } \\
\text { coverage (\%) }\end{array}$ \\
\hline ATR & gi 179161 & $53.05 / 6.32$ & $12(24)$ & 79 & 33 \\
CLU & gi 338305 & $37.00 / 5.74$ & $7(13)$ & 60 & 26 \\
LRG & gi 72059 & $34.55 / 5.66$ & $8(16)$ & 69 & 29 \\
ZAG & gi 7246026 & $31.86 / 5.70$ & $14(22)$ & 135 & 54 \\
KNG $^{c}$ & gi 125507 & $71.95 / 6.34$ & $9^{\mathrm{d})}$ & 434 & 19 \\
\hline
\end{tabular}

a) Spot ID are as in Fig. 1. $M_{\mathrm{r}}$ : nominal mass.

b) Accession numbers are from the MASCOT database (www.matrixscience.com).

c) Identified by LC-MS/MS analysis.

d) Number of peptides matched.

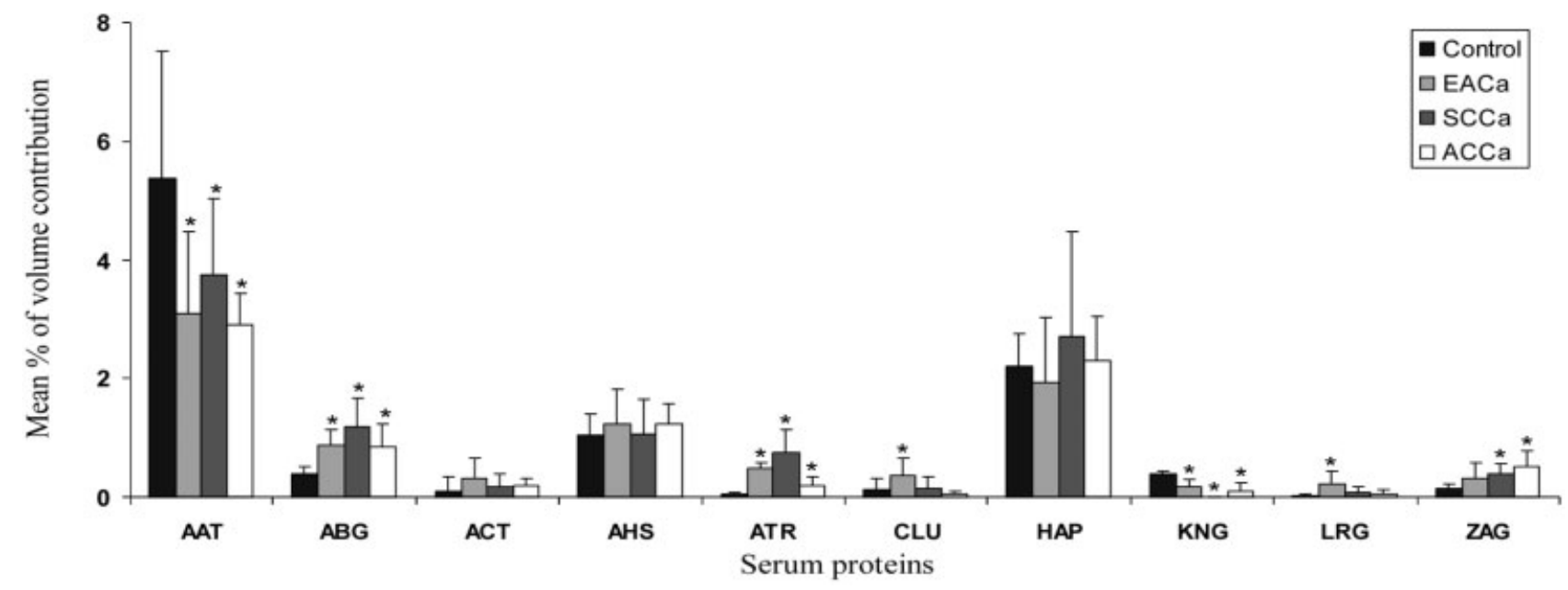

Figure 2. Mean percentage of volume contribution of 2-DE-resolved serum proteins. Volumes of protein spots were analyzed using the Image Master 2D-Platinum software, version 5.0. Analysis was restricted to ten clusters of protein spots including AAT, ABG, ACT, AHS, ATR, CLU, HAP, KNG, LRG and ZAG. Asterisks (*) denote significantly different values.

Table 2. Relative expression of serum proteins analyzed by 2-DE and ELISA

\begin{tabular}{|c|c|c|c|c|c|c|}
\hline \multirow[t]{2}{*}{$\begin{array}{l}\text { Serum } \\
\text { proteins }\end{array}$} & \multicolumn{3}{|c|}{$\begin{array}{l}\text { Analysis by } 2-\mathrm{DE} \\
\text { Fold changes relative to that of control }\end{array}$} & \multicolumn{3}{|c|}{$\begin{array}{l}\text { Analysis by ELISA } \\
\text { Fold changes relative to that of control }\end{array}$} \\
\hline & EACa patients & SCCa patients & ACCa patients & EACa patients & SCCa patients & ACCa patients \\
\hline AAT & $-0.6(p=0.005)$ & $-0.7(p=0.005)$ & $-0.5(p=0.0003)$ & $-0.4(p=0.0001)$ & $-0.5(p=0.006)$ & $-0.3(p=0.0008)$ \\
\hline$A B G$ & $+2.2(p=0.0001)$ & $+3.1(p=0.0001)$ & $+2.2(p=0.002)$ & $+1.6(p=0.0001)$ & $+1.6(p=0.001)$ & $+1.4(p=0.003)$ \\
\hline ACT & n.s. & n.s. & n.s. & n.d. & n.d. & n.d. \\
\hline AHS & n.s. & n.s. & n.s. & n.d. & n.d. & n.d. \\
\hline ATR & $+10.3(p=0.0001)$ & $+15.6(p=0.0001)$ & $+4.1(p=0.01)$ & $+2.3(p=0.0001)$ & $+2.2(p=0.0001)$ & $+2.2(p=0.0001)$ \\
\hline CLU & $+3.3(p=0.002)$ & n.s. & n.s. & $+1.0(p=0.005)$ & n.s. & n.s. \\
\hline HAP & n.s. & n.s. & n.s. & n.d. & n.d. & n.d. \\
\hline KNG & $-0.5(p=0.0001)$ & $-0.01(p=0.0001)$ & $-0.3(p=0.002)$ & n.d. & n.d. & n.d. \\
\hline LRG & $+10.9(p=0.01)$ & n.s. & n.s. & n.d. & n.d. & n.d. \\
\hline ZAG & n.s. & $+2.6(p=0.0003)$ & $+3.5(p=0.0001)$ & n.d. & n.d. & n.d. \\
\hline
\end{tabular}

+ , Increase in expression; -, decrease in expression; n.s., not significant and n.d., not determined.

The Student's $t$-test was used to analyze the significance of differences between normal subjects and patients. A $p$-value of less than 0.05 was considered significant. 

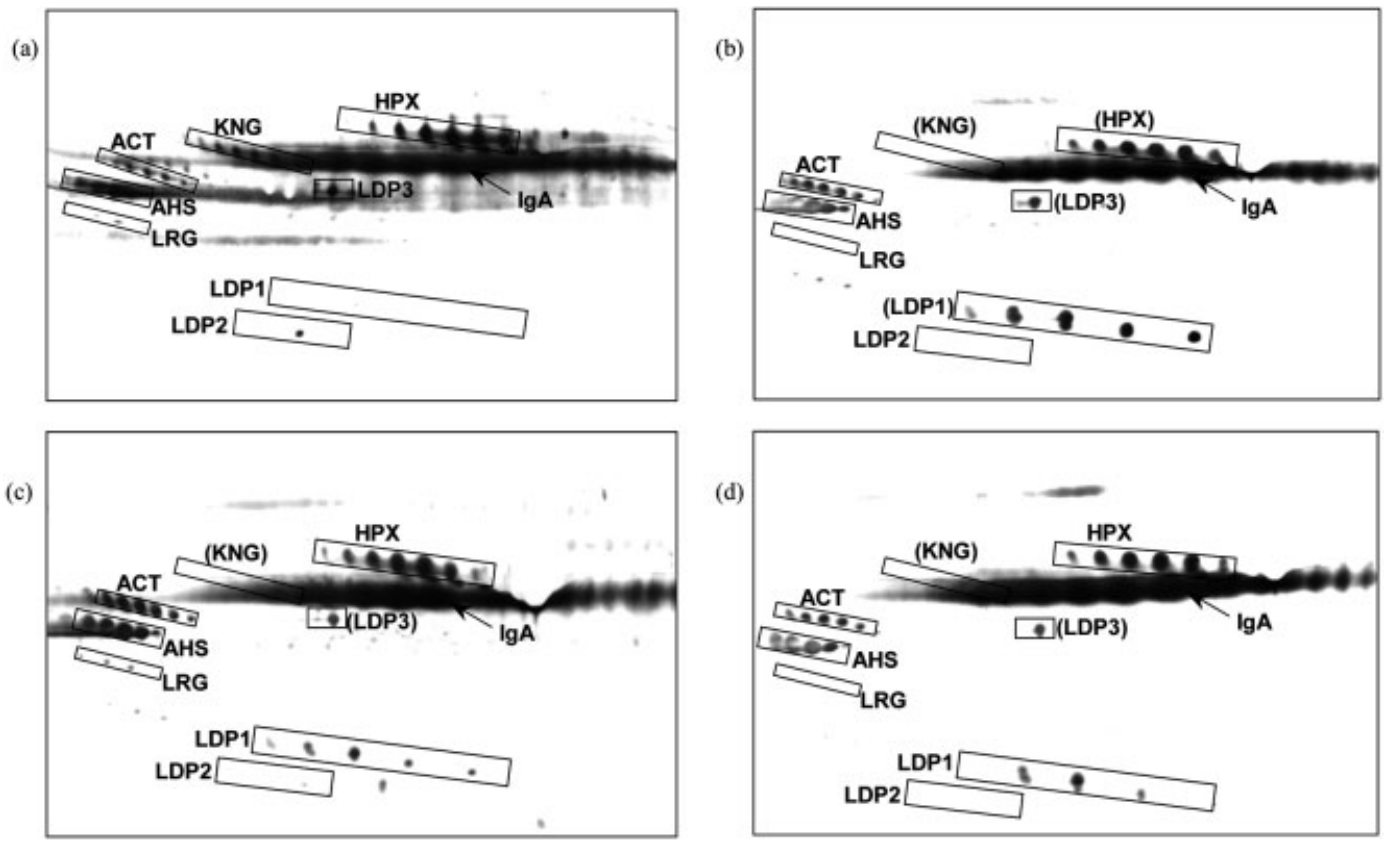

Figure 3. Analysis of $O$-glycosylated serum proteins by detection with CGB-lectin. Unfractionated serum samples of normal female controls (a), patients with EACa (b), patients with SCCa (c) and patients with ACCa (d), were subjected to 2-DE and lectin blotting. O-Glycosylated serum protein spots resolved included IgA, ACT, AHS, HPX, KNG and LRG and three unidentified glycoproteins termed, LDP1, LDP2 and LDP3. The aberrantly expressed proteins are marked in parentheses.

dance proteins in the respective cohorts of patients with EACa, SCCa and ACCa, relative to negative control women. The upregulated expression of ABG and ATR and downregulated expression of AAT and KNG were consistently observed in all the patients. However, CLU and LRG were upregulated only in the EACa patients, while patients with SCCa and ACCa were typically characterized by the upregulated expression of ZAG.

By using ELISA, the altered levels of ABG, ATR, AAT and CLU in the respective cohorts of the cancer patients were confirmed although the magnitude of fold differences were much lower compared to the 2-DE analysis. This is indicative of the higher sensitivity of 2-DE compared to ELISA when used to differentiate the levels of serum proteins.

Taken together, the altered patterns of expression of highabundance proteins detected for EACa, SCCa and ACCa were different when compared to our previously reported protein expression profiles of patients with BrCa and NPCa [13, 14], although the upregulated expression of CLU and downregulated expression of AAT have also been observed for the BrCa patients.

Probable reasons for the altered expression of CLU and AAT in cancer patients have been discussed in our previous report on $\mathrm{BrCa}$ [13]. The association of $\mathrm{ABG}$ and LRG, secretory proteins of unknown function [21, 22], with cancer has not been reported. Nevertheless, heavily glycosylated membrane proteins, with regions containing leucine-rich repeats similar to LRG, have been shown to be overexpressed in premalignant mammary epithelial cells [23] and BrCa tissues [24].

ATR is an important serine protease inhibitor in the plasma that is involved in the regulation of the blood coagulation cascade [25]. Differential levels of serum ATR in patients with EACa, SCCa and ACCa have not been previously reported although the increased levels of ATRthrombin complex have been demonstrated in the plasma of lung cancer patients [26] as well as patients with the various gynecological cancers [27]. Among the latter, the highest levels of ATR-thrombin complex were detected in patients with ovarian carcinoma, followed by cervical cancer patients and endometrial cancer patients, respectively [27]. Increased ATR detected in the sera of EACa, SCCa and ACCa patients in the present study may or may not have been part of such complex. However, this could not be verified using the approach in the present study as thrombin, which consists of 6 and $31 \mathrm{kDa}$ subunits, could not be detected within the resolved range of our 2-DE.

In the case of ZAG, previous reported studies have also demonstrated overexpression of the protein in sera of patients with prostate carcinoma [28] and $\mathrm{BrCa}$ [29]. Although we have not detected the expression of ZAG in our previous studies on BrCa patients [13], higher expression of the protein was clearly observed in all 2-DE serum protein profiles of patients with SCCa and ACCa in the present study. To the best of our knowledge, there had been no previous report on ZAG expression in patients with gynecological cancer. ZAG, named due to its tendency to precipitate zinc 


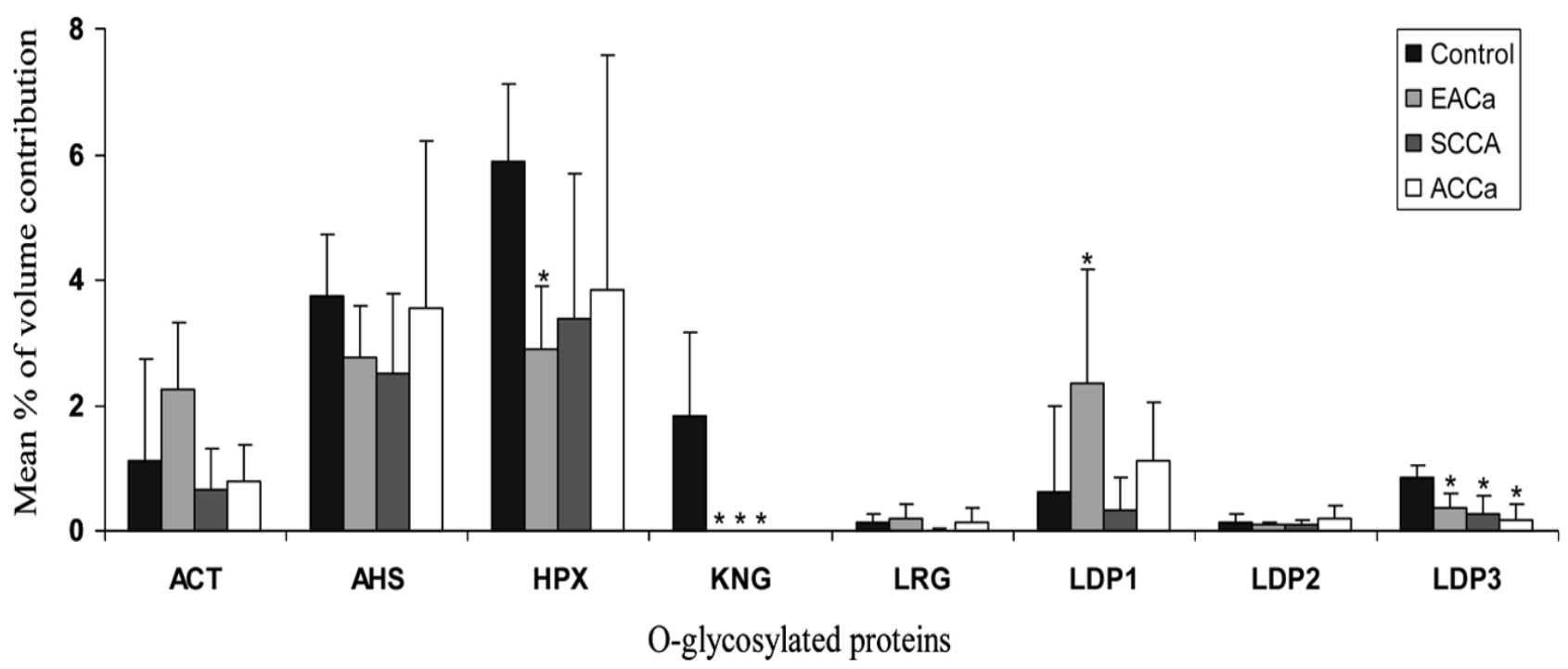

Figure 4. Mean percentage of volume contribution of CGB-lectin detected serum $O$-glycosylated proteins. Volumes of $O$-glycosylated protein spots were analyzed using Image Master 2D-Platinum software, version 5.0. Analyses were restricted to eight clusters of protein spots including ACT, AHS, HPX, KNG and LRG and unidentified glycoproteins, LDP1, LDP2 and LDP3. Asterisks (*) denote significantly different values.

salts and also known as $\alpha-2$ glycoprotein, is secreted by a variety of normal epithelia [30]. It stimulates lipolysis of adipocytes [31], and thus, may be involved in the development of cancer cachexia.

The use of enzyme-conjugated lectin to detect 2-DE separated O-glycosylated proteins further confirmed comparable expression of AHS and ACT between patients and negative control women as well as the patients' downregulated expression of KNG. KNG, an O-glycosylated serum polypeptide, is the light chain component of cleaved highmolecular-weight kininogen (HKa). HKa is known for its antiangiogenic properties as well as its inhibitory action on the proliferation of endothelial cells [32]. Our data suggest that the sera of patients with EACa, SCCa and ACCa were physiologically depleted of KNG. The disappearance of KNG from patients' sera was probably due to involvement of $\mathrm{HKa}$ in mechanisms to counter cancer-related angiogenesis and cell proliferation. A similar reduction in KNG expression was also observed in sera of patients with breast cancer (previously reported as unidentified protein, PR3 [13]), while reduced levels of high-molecular-weight kininogen have been detected in the plasma of patients with gastrointestinal cancer [33].

In addition to its use in validating the 2-DE data, the lectin approach provided an extra avenue to determine expression of additional high-abundance serum proteins that were either not detected or not well resolved using the silver staining method. Reduced levels of LDP3 in all three cohorts of patients and different altered levels of LDP1 and HPX specifically in patients with EACa were further shown. However, the expression of LRG was not significantly differ- ent between all cohorts of patients and negative control women. This is contrary to the earlier data that demonstrated significantly higher expression of LRG in EACa patients using silver staining. The different results may be resolved by increasing the sampling size of patients and controls in both experiments.

The marked difference in the intensities of LDP1 detected between EACa patients and control women using enzymeconjugated CGB lectin is perhaps more intriguing. Since this disparity was not detected in the earlier studies by silver staining, there is a strong possibility that the protein may be differently glycosylated. This is not exactly unexpected since the CGB lectin binds to the O-linked oligosaccharides [18] which contain core Galß1-3GalNAc structure. The disaccharide moiety, also known as the Thomsen-Friedenreich (TF) antigen, is one of the carbohydrate tumor antigens of the cell membrane [34]. It is cryptically present in O-glycans of a variety of membrane glycoproteins and becomes exposed during transition to malignancy [35]. In the present study, this is reflected in the secretory serum protein, LDP1, which demonstrated weak interaction with the CGB lectin in negative control women but appeared to react strongly with the lectin in patients with EACa. Because of the difference in carbohydrate structures between LDP1 in EACa patients compared to control women, the serum glycoprotein is a likely candidate as a serological marker for diagnosis of the cancer. However, the identity of LDP1 (as well as LDP2 and LDP3) was not possibly determined using the present methods of MS. Visual comparison of the 2-DE serum protein profiles with the Swiss ExPASy profiles also failed to detect any precise match with the annotated plasma proteins. 
We thank Ms. Noor Faezah Paizan for preparation and quality analysis of the CGB lectin. This work was funded by IRPA grants 06-02-03-1005 and 12-02-03-2021, from the Ministry of Science, Technology and Innovation, Malaysia. Protein MS/MS analysis has been facilitated by access to the Australian Proteome Analysis Facility established under the Australian Government's Major National Research Facilities Program.

\section{References}

[1] Cheah, P. L., Looi, L. M., Malays. J. Pathol. 1999, 21, 1-15.

[2] Gadducci, A., Cosio, S., Carpi, A., Nicolini, A., Genazzani, A. R., Biomed. Pharmacother. 2004, 58, 24-38.

[3] Niloff, J. M., Klug, T. L., Schaetzl, E., Zurawski, V. R., Jr. et al., Am. J. Obstet. Gynecol. 1984, 148, 1057-1058.

[4] Ginath, S., Menczer, J., Fintsi, Y., Ben-Shem, E. et al., Int. J. Gynecol. Cancer 2002, 12, 372-375.

[5] Borras, G., Molina, R., Xercavins, J., Ballesta, A., Iglesias, J., Gynecol. Oncol. 1995, 57, 205-211.

[6] Body, J. J., Sculier, J. P., Raymakers, N., Paesmans, M. et al., Cancer 1990, 65, 1552-1556.

[7] Molina, R., Torres, M. D., Moragas, M., Perez-Villa, J. et al., Tumour Biol. 1996, 17, 81-89.

[8] Kimura, Y., Fujieda, S., Takabayashi, T., Tanaka, T. et al., Cancer Lett. 2000, 155, 163-168.

[9] Verma, M., Wright, G. L., Jr., Hanash, S. M., Gopal-Srivastava, R., Srivastava, S., Ann. N. Y. Acad. Sci. 2001, 945, 103115.

[10] Wulfkuhle, J. D., Paweletz, C. P., Steeg, P. S., Petricoin, E. F., III, Liotta, L., Adv. Exp. Med. Biol, 2003, 532, 59-68.

[11] Veenstra, T. D., Conrads, T. P., Hood, B. L., Avellino, A. M. et al., Mol. Cell. Proteomics 2005, 4, 409-418.

[12] Gianazza, E., Eberini, I., Villa, P., Fratelli, M. et al., J. Chromatogr. B 2002, 771, 107-130.

[13] Doustjalali, S. R., Yusof, R., Yip, C. H., Looi, L. M. et al., Electrophoresis 2004, 25, 2392-2401.

[14] Doustjalali, S. R., Yusof, R., Govindasamy, G. K., Bustam, A. Z. et al., J. Med. Invest. 2006, 53, 20-28.

[15] Yong, P. H., Junit, S. M., Harun, F., Hashim, O. H., Clin. Biochem. 2006, 39, 126-132.
[16] Heukeshoven, J., Dernick, R., Electrophoresis 1988, 9, 28-32.

[17] Sanchez, J. C., Appel, R. D., Golaz, O., Pasquali, C. et al., Electrophoresis 1995, 16, 1131-1151.

[18] Abdul Rahman, M., Karsani, S., Othman, I., Abdul Rahman, P., Hashim, O. H., Biochem. Biophys. Res. Commun. 2002, 295, 1007-1013.

[19] Hashim, O. H., Gendeh, G. S., Jaafar, M. I., Biochem. Mol. Biol. Int. 1993, 29, 69-76.

[20] Hashim, O. H., Ng, C. L., Gendeh, S., Nik Jaafar, M. I., Mol. Immunol. 1991, 28, 393-398.

[21] Ishioka, N., Takahashi, N., Putnam, F. W., Proc. Natl. Acad. Sci. USA 1986, 83, 2363-2367.

[22] O’Donnell, L. C., Druhan, L. J., Avalos, B. R., J. Leukoc. Biol. $2002,72,478-485$.

[23] Carsberg, C. J., Myers, K. A., Stern, P. L., Int. J. Cancer 1996, 68, 84-92.

[24] Leygue, E., Snell, L., Dotzlaw, H., Troup, S. et al., J. Pathol. 2000, 192, 313-320.

[25] Potempa, J., Korzus, E., Travis, J., J. Biol. Chem. 1994, 269, 15957-15960.

[26] Tricerri, A., Vangeli, M., Errani, A. R., Guidi, L. et al., Oncology 1996, 53, 455-460.

[27] Gadducci, A., Marrai, R., Baicchi, U., Gagetti, O. et al., Gynecol. Oncol. 1996, 61, 215-217.

[28] Hale, L. P., Price, D. T., Sanchez, L. M., Demark-Wahnefried, W., Madden, J. F., Clin. Cancer Res. 2001, 7, 846-853.

[29] Bundred, N. J., Scott, W. N., Davies, S. J., Miller, W. R., Mansel, R. E., Eur. J. Cancer 1991, 27, 549-552.

[30] Burgi, W., Schmid, K., J. Biol. Chem. 1961, 236, 1066-1074.

[31] Hirai, K., Hussey, H. J., Barber, M. D., Price, S. A., Tisdale, M. J., Cancer Res. 1998, 58, 2359-2365.

[32] Colman, R. W., Jameson, B. A., Lin, Y., Johnson, D., Mousa, S. A., Blood 2000, 95, 543-550.

[33] Roeise, O., Sivertsen, S., Ruud, T. E., Bouma, B. N. et al., Cancer 1990, 65, 1355-1359.

[34] Springer, G. F., J. Mol. Med. 1997, 75, 594-602.

[35] Goletz, S., Cao, Y., Danielczyk, A., Ravn, P., in: Axford, J. S. (Ed.), Glycobiology and Medicine, Kluwer Academic/Plenum Publishers, New York 2003, pp. 147-162. 